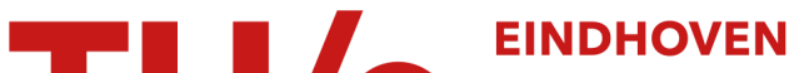 \\ UNIVERSITY OF \\ TECHNOLOGY
}

\section{Shape and director-field transformation of tactoids}

\section{Citation for published version (APA):}

Prinsen, P., \& Schoot, van der, P. P. A. M. (2003). Shape and director-field transformation of tactoids. Physical Review E - Statistical, Nonlinear, and Soft Matter Physics, 68(2), 021701-1/11. [021701].

https://doi.org/10.1103/PhysRevE.68.021701

DOI:

10.1103/PhysRevE.68.021701

Document status and date:

Published: 01/01/2003

\section{Document Version:}

Publisher's PDF, also known as Version of Record (includes final page, issue and volume numbers)

\section{Please check the document version of this publication:}

- A submitted manuscript is the version of the article upon submission and before peer-review. There can be important differences between the submitted version and the official published version of record. People interested in the research are advised to contact the author for the final version of the publication, or visit the $\mathrm{DOI}$ to the publisher's website.

- The final author version and the galley proof are versions of the publication after peer review.

- The final published version features the final layout of the paper including the volume, issue and page numbers.

Link to publication

\section{General rights}

Copyright and moral rights for the publications made accessible in the public portal are retained by the authors and/or other copyright owners and it is a condition of accessing publications that users recognise and abide by the legal requirements associated with these rights.

- Users may download and print one copy of any publication from the public portal for the purpose of private study or research.

- You may not further distribute the material or use it for any profit-making activity or commercial gain

- You may freely distribute the URL identifying the publication in the public portal.

If the publication is distributed under the terms of Article 25fa of the Dutch Copyright Act, indicated by the "Taverne" license above, please follow below link for the End User Agreement:

www.tue.nl/taverne

Take down policy

If you believe that this document breaches copyright please contact us at:

openaccess@tue.nl

providing details and we will investigate your claim. 


\title{
Shape and director-field transformation of tactoids
}

\author{
Peter Prinsen and Paul van der Schoot* \\ Polymer Physics Group, Department of Applied Physics and Dutch Polymer Institute, Eindhoven University of Technology, \\ P.O. Box 513, 5600 MB Eindhoven, The Netherlands
}

(Received 18 September 2002; revised manuscript received 2 April 2003; published 6 August 2003)

\begin{abstract}
Tactoids are droplets of a nematic phase that under suitable conditions form in dispersions of elongated colloidal particles. We theoretically study the shape and the director-field configuration of such droplets for the case where a planar anchoring of the director field to the interface is favored. A minimum of four regimes can be identified in which the droplets have a different structure. Large droplets tend to be nearly spherical with a director field that is bipolar if the surface tension is strongly anisotropic and homogeneous if this is not so. Small droplets can become very elongated and spindlelike if the surface tension is sufficiently anisotropic. Depending on the anchoring strength, the director field is then either homogeneous or bipolar. We find that the more elongated the tactoid, the more strongly it resists the crossing over from a homogeneous to a bipolar structure. This should have implications for the nucleation rate of the nematic phase. Our calculations qualitatively describe the size dependence of the aspect ratio of tactoids found in recent experiments.
\end{abstract}

DOI: 10.1103/PhysRevE.68.021701

PACS number(s): 61.30.Pq, 61.30.Dk, 82.70.-y

\section{INTRODUCTION}

The spindle-shaped droplets that are commonly referred to as tactoids form when a uniaxial nematic liquid-crystalline phase is nucleated out of an isotropic fluid dispersion containing highly anisometric colloidal particles. They have long been observed in dispersions of tobacco mosaic virus, $f d$ virus, vanadium pentoxide, aluminum oxyhydroxide, iron oxyhydroxide, tungsten oxide, and so forth [1-4]. (For an historical overview, the reader is referred to a recent review paper by Sonin [5].) Bernal and Fankuchen provided a plausible rationalization of the remarkable shape of the tactoids in terms of an anisotropic interfacial tension between the coexisting isotropic and nematic phases [1]. The equilibrium shape of nematic liquid-crystalline drops with a fixed, homogeneous director field was considered in more detail by Herring [6], Chandrasekhar [7], and later Virga [8]. These works show that homogeneous nematic drops should not only be elongated but in addition develop sharp ends if the anisotropy of the surface tension is sufficiently large. However, the idea that the shape typical of tactoids is due to an anisotropic surface tension has not become generally accepted, not least because it fails to explain the dependence of the aspect ratio of the nematic droplets upon their size [7].

The current paradigm for the tactoid structure involves not a homogeneous but a bipolar director field [5], a consequence of the circumstance that elongated colloidal particles for entropic reasons tend to align parallel the isotropicnematic interface [9-12]. In the bipolar configuration, the director field smoothly follows the contour of the surface of the droplet, connecting two diametrically opposed point defects called boojums on the surface of the droplet [13]. In a way, the director field radiates outward from one boojum to converge again on the other as is depicted in Fig. 1. Polarization microscopic images taken of tactoids in dispersions

\footnotetext{
*Corresponding author.

Email address: p.vanderschoot@phys.tue.nl
}

of tobacco mosaic virus [1], aluminum oxyhydroxide [2], and vanadium pentoxide [14] do indeed very strongly suggest these to be bipolar.

The precise shape and structure of bipolar tactoids was studied by Williams [15], who solved by numerical methods the Euler-Lagrange equations obtained for the (idealized) case where the three bulk elastic constants of the director field are equal, and the anchoring is tangential everywhere on the surface of the droplet. The calculations show that bipolar tactoids optimize their shape by striking a balance between a minimal deformation of the director field and a minimal interfacial area. For large bipolar drops, the interfacial free energy dominates so these tend to be (nearly) spherical. Small drops reduce their elastic deformation by attaining an elongated shape because these are dominated by the stiffness of the director field. We deduce from the work of Williams [15] that the (gradual) crossover from a spherical to a highly elongated shape occurs when the dimensionless ratio $\kappa \equiv K / \tau V^{1 / 3}$ exceeds a value of about unity, because then the bulk elasticity takes over from the surface energy. Here, $K$ denotes a Frank elastic constant, $\tau$ is the interfacial tension of the coexisting phases, and $V$ is the volume of the droplet. Typical values for the elastic constants and the interfacial tension are $K \approx 10^{-13}-10^{-11} \mathrm{~N}[16-23]$ and $\tau$ $\approx 10^{-7}-10^{-5} \mathrm{~N} \mathrm{~m}^{-1}$ [24-27], so tactoids of a linear dimension in the micrometer range should be elongated, not spherical. This seems to be borne out by observation [5].

A caveat of the calculations of Williams is the condition of tangential alignment of the director field, which cannot be valid if the drops are, in some sense, small [8]. In fact, one can show by a straightforward scaling argument [28] that for spherical droplets a bipolar director field, as taken by Williams [15], must be less stable than a homogeneous director field, assumed in Refs. [6-8], if $\kappa$ is larger than a dimensionless surface anchoring strength $\omega$. This anchoring strength is small if the interfacial tension is isotropic or nearly so, and large if it is strongly anisotropic and parallel alignment of the director field is favored. For uncharged, slender colloids $\omega$ has been predicted to have a value around 
unity [9-12], so in many practical situations the shape and director-field transformations are not independent. Recent calculations on almost spherical nematic droplets indicate that this must indeed be so $[29,30]$.

Despite considerable progress in recent years [13], the structure of nematic droplets, in general, and of tactoids, in particular, remains incompletely understood. In our view, the problem merits a closer inspection both from an experimental and a theoretical point of view. In this work, we present a theoretical study of the tactoid surface shape and directorfield structure, and focus on the influence of quantities such as the volume of the droplets, the Frank elastic constants, and the degree of anisotropy of the surface tension. Our aim is to connect the early interpretation of the tactoid shape based on an anisotropic surface tension [6,7], with the current view, which focuses on the response of the droplet shape to the bipolar director field [15]. As we shall see, the minimum anchoring strength required to force a homogeneous director field to become bipolar depends both on the volume and on the shape of the droplet, with elongated droplets resisting a bipolar director field much more strongly than spheroidal ones. This could have far-reaching consequences for the nucleation kinetics of lyotropic nematic phases.

The remainder of this paper is structured as follows. In Sec. II, we present a phenomenological free energy functional, in which we combine an elastic energy associated with the deformation of the director field with an anisotropic interfacial free energy. The former consists of the usual contributions from the splay, the twist, the bend, and the saddlesplay distortions, and the latter of a sum of an isotropic surface free energy and a free energy penalizing a nonplanar anchoring of the director field. Arguments shall be given in favor of (i) the approximation of equal elastic constants and (ii) the neglect of the contribution from the saddle-splay deformation. Next, in Sec. III, a scaling estimate of the optimal droplet configuration is presented. For reasons of computational simplicity, we focus on spherical and highly elongated droplet shapes for two extreme director fields, one purely homogeneous and one purely bipolar. In Sec. IV, we calculate the free energy for three different droplet shapes and director-field configurations exactly. The main result of our calculations constitutes a "phase" diagram for the equilibrium shape and director-field configuration of the droplets as a function of the elastic stiffness of the director field and the anisotropy of the interfacial tension.

We end this paper with a discussion in Sec. V, where we compare our theory with experimental data and where we propose a continuous crossover mechanism from the homogeneous to the bipolar director-field configuration.

\section{FREE-ENERGY FUNCTIONAL}

We consider a nematic droplet of volume $V$ in an isotropic, fluid medium. If the volume of the droplet is macroscopic on the scale of the nematogens, we need not take into account finite-size effects on the stability of the nematic and on the degree of nematic order in the droplet [29,31]. The free energy $F$ of a nematic tactoid can then be written as the sum of the free-energy cost of a potential deformation of the director field $F_{E}$ and that of the presence of the interface with the isotropic medium $F_{S}$

$$
F=F_{E}+F_{S} .
$$

For the interfacial free energy, we adopt the following plausible expression [32]:

$$
F_{S}=\tau \int_{A} d^{2} \mathbf{r}\left[1+\omega(\mathbf{q} \cdot \mathbf{n})^{2}\right],
$$

with $\mathbf{n}=\mathbf{n}(\mathbf{r})$ the nematic director and $\mathbf{q}=\mathbf{q}(\mathbf{r})$ the surface normal at the position $\mathbf{r}$ on the interface between the droplet and the medium; $\tau$ is the interfacial tension and $\omega$ is the earlier-introduced dimensionless anchoring strength. We set $\omega>0$ to ensure that a parallel anchoring of the director field to the interface is favored [8]. The integration in Eq. (2) is over the entire interfacial area $A$ of the droplet. Although $a d$ $h o c$, our choice for the interfacial free energy fairly accurately mimics the anchoring-angle dependence of various theoretical predictions for hard rods and hard worms [9-12].

The (Frank) elastic free energy $F_{E}$ takes the form [33-35]

$$
\begin{aligned}
F_{E}= & \int_{V} d^{3} \mathbf{r}\left[\frac{1}{2} K_{1}(\boldsymbol{\nabla} \cdot \mathbf{n})^{2}+\frac{1}{2} K_{2}(\mathbf{n} \cdot \boldsymbol{\nabla} \times \mathbf{n})^{2}\right. \\
& \left.+\frac{1}{2} K_{3}[\mathbf{n} \times(\boldsymbol{\nabla} \times \mathbf{n})]^{2}-K_{24} \boldsymbol{\nabla} \cdot[\mathbf{n} \boldsymbol{\nabla} \cdot \mathbf{n}+\mathbf{n} \times(\boldsymbol{\nabla} \times \mathbf{n})]\right],
\end{aligned}
$$

where $\mathbf{n}=\mathbf{n}(\mathbf{r})$ again denotes the locally defined director, and the integration is over the entire volume $V$ of the droplet. The first three terms, with the elastic constants $K_{1}, K_{2}$, and $K_{3}$, represent the usual splay, twist, and bend deformation modes of a bulk nematic [36]. The last term, with the elastic constant $K_{24}$, stems from the saddle-splay deformation mode. For this term, the volume integral can be transformed to a surface integral, which is why it is usually ignored in studies of bulk nematics. Since our droplets have a large surface-to-volume ratio, it is not a priori clear that the "surface" elastic term can indeed be neglected.

Obviously, Eq. (3) needs to be simplified for the theory to be practical. To make headway, we first note that a (paritybroken) twisted director-field configuration has [13], as far as we are aware, not (yet) been observed in lyotropic (colloidal) systems. That this is not entirely unexpected is discussed in more detail in Sec. V. Hence, we ignore the possibility of a twisted bipolar configuration and drop from our free energy the second (twist-deformation) term. Further simplification follows from the observation that for the bispherical director field advanced by Williams in Ref. [37] and described in more detail in Sec. IV, the saddle-splay deformation merely renormalizes the contribution of the splay elastic deformation to the free energy. (See also Appendix B.) We therefore absorb the influence of $K_{24}$ into $K_{1}$ and drop the saddle-splay term also. Note that little is known about the magnitude of the surface elastic constant $K_{24}$ of lyotropic nematics of elongated colloids. 
a)

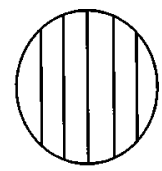

c)

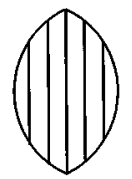

b)

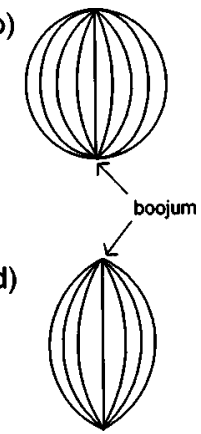

FIG. 1. Schematic representation of some of the nematic droplet shapes and structures discussed in the main text. Top: spherical droplets with (a) a homogeneous and (b) a bipolar director field. Bottom: spindle-shaped tactoids with (c) a homogeneous and (d) a bipolar director field. Indicated also are the locations on the poles of the droplets of the surface point defects ("boojums").

This leaves us with two elastic constants, $K_{1}$ and $K_{3}$, relevant to the problem in hand. From theoretical work we infer that $K_{3} \gg K_{1}$ if the colloidal particles behave like rigid rods and that $K_{3} \approx K_{1}$ if they are better described as semiflexible, wormlike chains [16,40-43]. Experimental data on dispersions of, e.g., the rodlike tobacco mosaic virus and the semiflexible polymer poly(butyl glutamate) agree qualitatively with these predictions $[16,41]$. This would suggest that the commonly used equal-constant approximation $K=K_{1}$ $=K_{3}$ is of limited use for nematics of rodlike colloids, except if they are not extremely rigid. However, the equalconstant approximation may, in fact, be quite reasonable for stiff colloids too because the splay elastic term overwhelmingly dominates the overall elastic free energy of a bipolar droplet [37]. (See also Sec. III.) For this reason, we adopt the equal-constant approximation too and write

$$
F_{E}=\frac{1}{2} K \int_{V} d^{3} \mathbf{r}\left\{(\boldsymbol{\nabla} \cdot \mathbf{n})^{2}+[\mathbf{n} \times(\boldsymbol{\nabla} \times \mathbf{n})]^{2}\right\}
$$

A discussion of the impact of unequal elastic constants can be found in Sec. V.

\section{SCALING THEORY}

We first analyze the free-energy functionals Eqs. (2) and (4) from a scaling theoretical point of view, and obtain a qualitative picture of the equilibrium droplet shapes and structures based on the four extreme cases of Fig. 1. These are the cases of (i) elongated bipolar drops, (ii) spherical bipolar drops, (iii) elongated homogeneous drops, and (iv) spherical homogeneous drops, which we discuss case by case [44]. Finally, at the end of this section, possible transitions between the various configurations are investigated by comparing their free energies.

(i) Elongated, bipolar drops. For cylindrically symmetric, bipolar drops with major axis $R$ and minor axis $r \ll R$, the interfacial free energy Eq. (1) obeys

$$
F_{S} \approx \tau R r
$$

because the total surface area $A$ is of the order of $R r$, and because $(\mathbf{q} \cdot \mathbf{n})^{2}=0$. The latter equality follows from our presumption of tangential anchoring, which by construction results in a free energy that is independent of the anchoring strength $\omega$. In Eq. (5), as in all of the following scaling estimates, all constants of proportionality are omitted.

To obtain a scaling estimate for the Frank elastic energy of the bipolar drops, we deduce from Eq. (4) that $F_{E}$ must be proportional to an elastic constant $K$ times a droplet volume $V \approx R r^{2}$ times the square of a reciprocal radius of curvature. By geometry, the mean radius of curvature of the bend deformation scales as $R^{2} / r$ and that of the splay deformation as $R$. This means that the contribution from the bend deformation can be neglected if the drops are highly elongated, that is, if $R \gg r$. (It can even be ignored if $R \approx r$ because a more precise calculation shows that for spherical drops the contribution from the bend deformation is 7.5 times smaller than that of the splay deformation irrespective of their size [37].) In conclusion,

$$
F_{E} \approx K R r^{2} \frac{1}{R^{2}}=K R\left(\frac{r}{R}\right)^{2}
$$

should be a reasonably accurate estimate for the free energy albeit, of course, within an unknown numerical prefactor. Equation (6) shows that the more elongated the droplet, the less nonuniform the director field and therefore, the smaller the free-energy penalty associated with the bipolar director field.

Ignoring the free energy of the microscopic core of the boojums, we obtain the optimal aspect ratio $R / r$ for a highly elongated droplet by minimizing (at a fixed droplet volume $V)$ the total free energy given by the sum of Eqs. (5) and (6),

$$
\frac{R}{r} \approx K^{3 / 5} \tau^{-3 / 5} V^{-1 / 5}=\kappa^{3 / 5} .
$$

Equation (7) implies that the aspect ratio of a bipolar drop is larger the smaller its volume. If we insert Eq. (7) into Eqs. (5) and (6), we find for the equilibrium free energy

$$
F \approx V^{3 / 5} \tau^{4 / 5} K^{1 / 5}=V^{2 / 3} \tau \kappa^{1 / 5},
$$

at least if $\kappa \gg 1$. Equations (7) and (8) are consistent with the more elaborate calculations of Williams [15].

(ii) Spheroidal, bipolar drops. In the opposite limit of $\kappa$ $\ll 1$, the drops are spheroidal and $R \approx r$. In this limit, we have by similar arguments

$$
F \approx K R+\tau R^{2}=V^{2 / 3} \tau(\kappa+1),
$$

as can in fact also be deduced directly from Eqs. (5) and (6) by setting $r=R$.

(iii) Elongated, homogeneous drops. If the director field is homogeneous, there is no elastic deformation so $F_{E}=0$. The surface free energy for the case $R \gg r$ becomes

$$
F_{S} \approx \tau R r\left[1+\omega\left(\frac{r}{R}\right)^{2}\right]
$$


because in that case $(\mathbf{q} \cdot \mathbf{n})^{2} \approx(r / R)^{2}$ for most of the interfacial area. The optimal aspect ratio of the highly elongated drops follows again from a free-energy minimization at constant volume, giving

$$
\frac{R}{r} \approx \omega^{1 / 2}
$$

which grows with increasing anchoring strength albeit not very strongly. The equilibrium free energy of the drops in the strong anchoring limit $\omega \gg 1$,

$$
F \approx V^{2 / 3} \tau \omega^{1 / 6}
$$

follows by insertion.

(iv) Spheroidal, homogeneous drops. Nematic droplets with a uniform director field are spheroidal if $0<\omega \ll 1$, so $R \approx r$ and $(\mathbf{q} \cdot \mathbf{n})^{2} \approx 1$. Hence, our estimate for the free energy reads

$$
F \approx V^{2 / 3} \tau(1+\omega)
$$

for this particular configuration.

The crossover from a homogeneous to a bipolar director field occurs if the surface anisotropy wins out from the elastic stiffness of the director field. According to the free-energy estimates, Eqs. (8), (9), (12), and (13), there cannot be a large jump in the aspect ratio of the drops at the point where the director field changes from homogeneous to inhomogeneous or vice versa. Therefore, spheroidal droplets remain spheroidal and elongated ones remain elongated.

For spheroidal droplets, the director-field transformation occurs if

$$
0<\omega \approx \kappa \ll 1,
$$

as can be deduced from Eqs. (9) and (13). By equating Eqs. (8) and (12), we find that in the opposite limit of elongated tactoids, the crossover from a homogeneous to a bipolar director field takes place when

$$
1 \ll \omega \approx \kappa^{6 / 5} .
$$

Equations (14) and (15) demarcate four regimes. (See also Fig. 6.) For $\kappa \gtrsim 1$ and $\omega \gtrsim 1$, the droplets are elongated, that is, tactoidal. These tactoids are homogeneous if $\omega \leq \kappa^{6 / 5}$ and bipolar if $\omega \geq \kappa^{6 / 5}$. For $\kappa \lesssim 1$ or $\omega \leqq 1$, the droplets are spheroidal with a homogeneous director field if $\omega \leq \kappa$ and spheroidal with a bipolar director field if $\omega \gtrsim \kappa$. Apparently, elongated tactoids postpone the crossover to a bipolar structure to higher droplet volumes than one would expect from the scaling behavior of the spherical ones. The reason is that the gain in surface free energy upon the replacement of a homogeneous director field by a bipolar director field is comparatively small if the nematic droplet is elongated.

\section{VARIATIONAL THEORY}

The theory presented in the preceding section has the merit of simplicity and of providing insight, but it lacks the ability to predict numerical prefactors and crossover details.
To go beyond simple scaling arguments, one would, ideally, minimize the free-energy functional $F=F[\mathbf{n}]$ with respect to the director field $\mathbf{n}(\mathbf{r})$ and the droplet shape (in some suitable parametrization of the surface). This would have to be done at a constant drop volume $V$ and at a constant $|\mathbf{n}| \equiv 1$ because the director is a vector of fixed unit length [36]. The resulting Euler-Lagrange equations are those of a free-surface problem, which are very difficult to solve even numerically $[8,15,30]$. We therefore simplify the mathematical problem (i) by prescribing certain families of (cylindrically symmetric) droplet shapes and director fields and (ii) by minimizing the free energy with respect to the pertinent variational parameters.

Here, we only briefly touch upon how the calculations were done and focus on the presentation of the results of the calculations. Readers interested in the details of the calculations are referred to Appendixes A and B. We first discuss our findings for the droplets with a homogeneous director field, and subsequently, those for the bipolar director field. We end this section with a comparison of the free energies of the two types of drop, allowing us to locate for what values of $\kappa$ and $\omega$ the transition from one to the other director field ought to be expected.

Homogeneous drops. For drops with a homogeneous director field, the minimization of the (surface) free energy can be done exactly with the help of the well-known Wulff construction $[6,7,45]$. Virga applied this procedure to a surface free energy of the form of Eq. (2) some time ago, but only very briefly discussed his findings. (See Ref. [8], and references cited therein.) We have repeated the calculations and for completeness present them in considerable detail in Appendix A. In agreement with the earlier work described in Ref. [8], we find the droplets to be spherical if $\omega \rightarrow 0$, elongated spheroidal if $0<\omega \leqslant 1$, and elongated with sharp ends (tactoidal) if $\omega>1$.

Expressions for the equilibrium free energy $\widetilde{F} \equiv F / \tau V^{2 / 3}$ obtained with the aid of the Wulff construction are given in Appendix A and plotted as a function of $\omega$ in Fig. 2. In the limit $\omega \rightarrow 0$, the free energy obeys $\widetilde{F} \sim 4.84+1.61 \omega$ to linear order in $\omega$, while it approaches the limiting relation $\widetilde{F}$ $\sim 5.66 \omega^{1 / 6}$ for $\omega \gg 1$, confirming our earlier estimates given by Eqs. (12) and (13).

For comparison, we have also calculated the optimal surface free energies of ellipses of revolution and of circle sections of revolution, and plotted the results in Fig. 2. (Details of the calculations can be found in Appendix A.) The figure shows that for all except very small values of $\omega$, the ellipses of revolution are of higher free energy than the circle sections of revolution that we for brevity refer to as "spindles" from now on. In the limit $\omega \rightarrow 0$, the differences between the free energies of the Wulff, spindle, and ellipsoid shapes are only of the order of $\omega^{2}$ and vanish rapidly with decreasing anchoring strength. Remarkably, the exact Wulff shapes and the spindles differ in free energy less than about one-tenth of a percent for all values of $\omega$.

The shape transformation of the nematic droplet with a homogeneous director field is illustrated in Fig. 3, where we have plotted the anchoring-strength dependence of the angle 


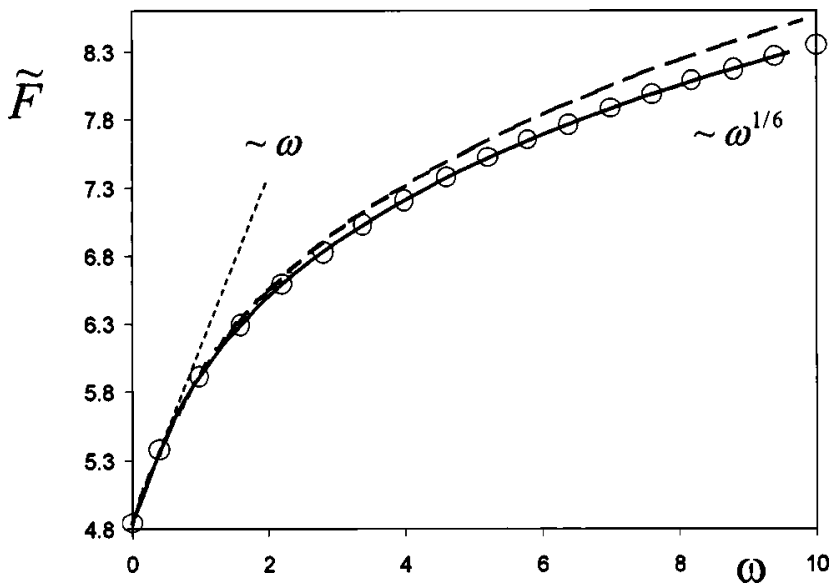

FIG. 2. Dimensionless free energy $\widetilde{F}$ of a nematic drop with a homogeneous director field as a function of the dimensionless anchoring strength $\omega$. Dashed line-ellipsoid of revolution, circlesWulff shape, drawn line-spindle. All curves approach the powerlaw scaling $\widetilde{F} \sim \omega^{1 / 6}$ in the limit $\omega \gg 1$. The initial slope for $\omega \rightarrow 0$ is identical for all three shapes.

between the main axis of the drop and the surface tangent taken at either pole (the tip angle). The Wulff construction gives a tip angle that is a constant $\pi / 2$ for $\omega \leqslant 1$, but that decreases as $\arctan 1 / \sqrt{\omega-1}$ if $\omega>1$. Also given in the figure are the tip angles for the prolate spheroids and the spindles, where we note that the former shape has a fixed tip angle of $\pi / 2 \simeq 1.57$. The tip angle of the spindle approaches the exact value from below in both limits $\omega \rightarrow 0$ and $\omega$ $\rightarrow \infty$.

Considering the relatively small differences in free energy, it is hardly surprising that the optimal aspect ratio of the droplets is quite insensitive to the precise droplet shape. The aspect ratio $R / r$ of the drops we obtained with the Wulff

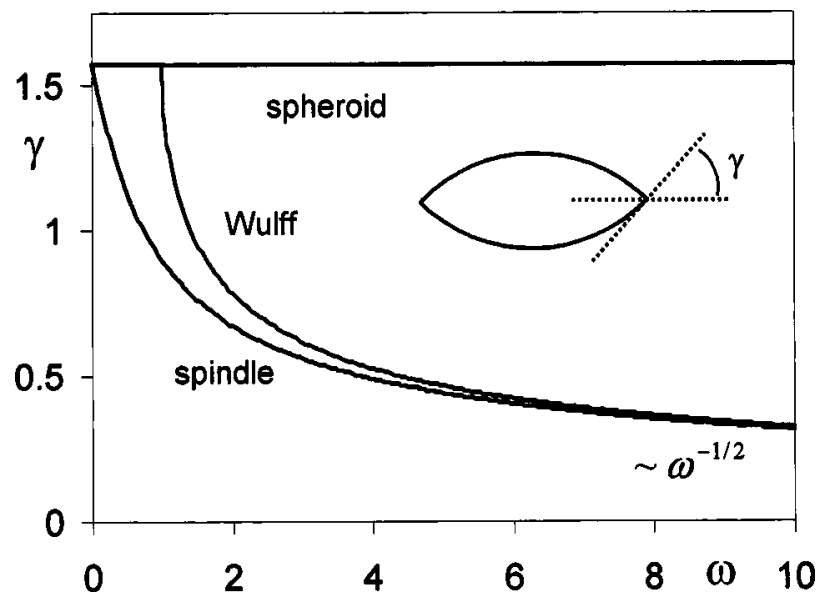

FIG. 3. The tip angle $\gamma$ of homogeneous drops (in radians) as a function of the dimensionless anchoring strength $\omega$ for the various shapes indicated. For $\omega \leqslant 1$, the Wulff shape predicts a spheroid shape with a tip angle of $\pi / 2 \approx 1.57$. For $\omega>1$, the Wulff shape has sharp ends with a tip angle smaller than $\pi / 2$. Both the spindle and the Wulff shape predict a scaling law $\gamma \sim \omega^{-1 / 2}$ for the tip angle at large $\omega$.

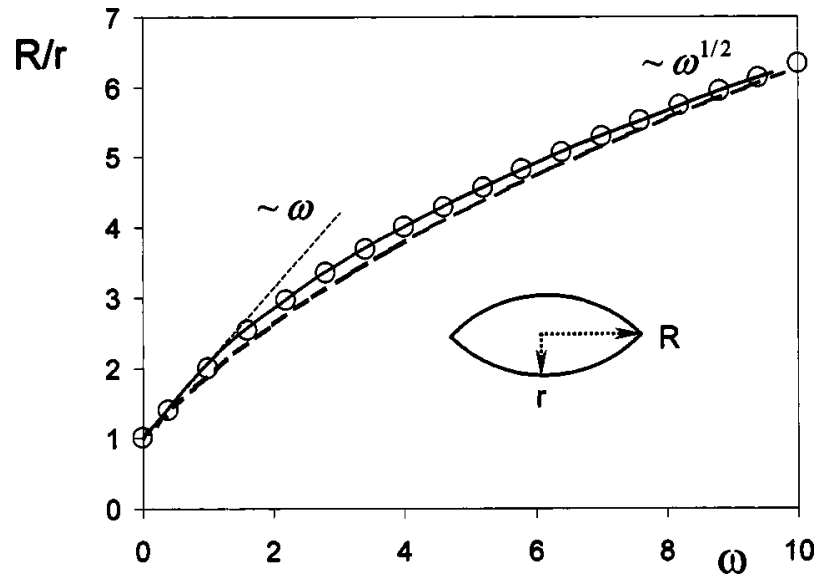

FIG. 4. Aspect ratio $R / r$ of homogeneous nematic drops as a function of the dimensionless anchoring strength $\omega$. Given are the results for the prolate spheroid, the spindle and the Wulff shape, indicated, respectively, by the dashed line, the drawn line, and the circles. The initial slope for $\omega \rightarrow 0$ is identical for all three shapes and so is the scaling $R / r \sim \omega^{1 / 2}$ for large $\omega$.

construction exactly obeys $R / r=1+\omega$ if $\omega \leqslant 1$ and $R / r$ $=2 \omega^{1 / 2}$ if $\omega>1$, quite close to the approximate results obtained with the prolate spheroids and the spindles. This is shown in Fig. 4, which also confirms that the aspect ratio of a homogeneous nematic drop is only a fairly weak function of the anchoring strength. In other words, large values of $\omega$ are required to get strongly elongated drops.

Bipolar drops. For tangentially anchored bipolar drops, the surface free energy is independent of $\omega$ and proportional to the total interfacial area as we pointed out earlier. At fixed volume, the total of the interfacial area is a function of the droplet shape. For the droplet shapes, we use two plausible ones, namely, ellipsoids of revolution and spindles [38]. The choice for the latter shape was inspired by optical micrographs of bipolar nematic droplets [2,5].

Williams noted in Ref. [37] that the director field of a director field of a spherical bipolar droplet is to a very good approximation bispherical, that is, tangent to circles of increasing radius of curvature, the closer we get to the central axis of the drop. The circles cross at the boojums on the poles of the droplet. (See Fig. 1.) It stands to reason that the director field of a bipolar spindle should also be approximately bispherical, which is what we now presume. (See also Ref. [39].) Similarly, the director field inside the ellipsoidal drop which we describe by nested ellipsoids of revolution that touch at the poles, following the ansatz of DuboisViolette and Parodi in their calculation of the elastic deformation energy of a spherical bipolar droplet [46].

Details of our calculations can be found in Appendix B. The dimensionless free energies $\widetilde{F}$ obtained for the bipolar ellipsoids and spindles are functions of $\kappa$ and of their aspect ratio $R / r$. These were minimized with respect to $R / r$, producing implicit expressions for the optimal aspect ratio of the drops that we could only solve by analytical methods in the limits $\kappa \rightarrow 0$ and $\kappa \rightarrow \infty$. For arbitrary values of $\kappa$, we solved the equations by standard numerical methods. Inserting the optimal values of $R / r$ back into $\widetilde{F}$ produced the equilibrium 


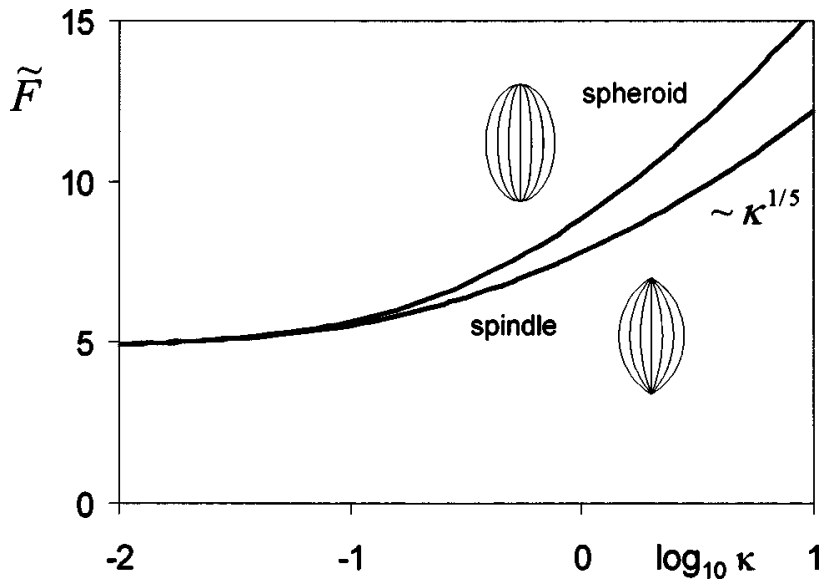

FIG. 5. Dimensionless free energy $\widetilde{F}$ of a bipolar tactoid as a function of the dimensionless elastic stiffness $\kappa=K / \tau V^{1 / 3}$. Indicated are results for prolate spheroids and for spindles. The free energy of the spindle shape is below that of the prolate spheroid shape for all $\kappa$. For large $\kappa$ the free energy of the bipolar spindle approaches the scaling form $\widetilde{F} \sim \kappa^{1 / 5}$. No pure scaling form is reached for the bipolar spheroid.

free energy for the two shapes.

In Fig. 5, we present the equilibrium free energy $\widetilde{F}$ as a function of the dimensionless elastic stiffness $\kappa$ for the ellipsoids and the spindles. The spindle shape resembling the experimentally found tactoid shape is indeed the lowest-freeenergy shape of the two for all values of $\kappa>0$. In the limit $\kappa \rightarrow 0$, where the droplets are spherical, we get for the bispherical director field $\widetilde{F} \sim 4.84+8.83 \kappa$ and for the ellipsoidal director field $\widetilde{F} \sim 4.84+9.35 \kappa$ (both to linear order in $\kappa$ ). Note that we correctly reproduce the results of Refs. [37], [46], valid in that limit.

For large $\kappa$, the free energy of the bipolar spindle approaches the scaling form $\widetilde{F} \sim 7.68 \kappa^{1 / 5}$, which confirms our scaling estimate Eq. (8). This is only slightly larger than the more accurate result $\widetilde{F} \sim 6.66 \kappa^{1 / 5}$ found by Williams (see Fig. 6 of Ref. [15]). The limiting form of the aspect ratio we find for the spindle reads $R / r \sim 4.44 \kappa^{3 / 5}$, the scaling exponent of which agrees with that of our estimate Eq. (7). Interestingly, neither the free energy nor the aspect ratio of the ellipsoids of revolution exhibit a pure power-law dependence in the limit of $\kappa \gg 1$.

Crossover. To determine which droplet structure is the most stable, we compare the free energies of the various droplet shapes and director-field configurations. For the droplet with the homogeneous director field, we use the shape obtained from the Wulff construction because it represents for that director-field configuration the shape of the lowest free energy. For the bipolar droplet, we take the spindle shape, which is more stable than the prolate spheroid irrespective of the magnitude of the elastic coupling parameter $\kappa$.

If we compare Fig. 2 with Fig. 5, we see that the homogeneous director field has a lower free energy than the bipolar director field if $\kappa$ is sufficiently large (corresponding to a sufficiently small droplet volume). For small $\kappa$ (large droplet

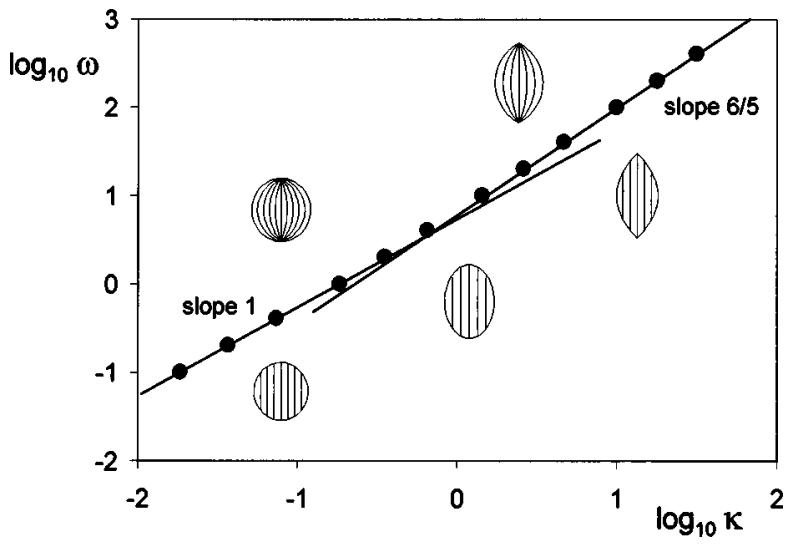

FIG. 6. Diagram of states of nematic tactoids as a function of the dimensionless elastic stiffness $\kappa$ and the dimensionless anchoring strength $\omega$. The filled circles indicate the crossover from a homogeneous to a bipolar director field according to our variational theory, and the lines emphasize the slopes 1 and $6 / 5$ obtained from the scaling estimate. Tactoid shape and director-field configuration are schematically given where they occur in the diagram of states.

volume), the reverse is true. The crossover value of $\kappa$, separating homogeneous and bipolar director-field configurations, depends on the magnitude of the anchoring strength $\omega$. This is shown in the "phase" diagram of Fig. 6. For clarity, the various director-field configurations and droplet shapes are schematically indicated in the figure roughly where they occur. Also indicated are the slopes to the points separating the regions corresponding to bipolar and homogeneous director fields for the nearly spherical and the highly elongated droplets. They are in complete agreement with the scaling results Eqs. (14) and (15), for which we can now also produce the prefactors: $\omega \sim 5.45 \kappa$ for $\omega \ll 1$ and $\omega \sim 6.24 \kappa^{6 / 5}$ for $\omega \gg 1$.

Finally, in Fig. 7, we give the calculated aspect ratio of the tactoids as a function of $\kappa$ for a bipolar director field, as that for a homogeneous director field with a fixed anchoring

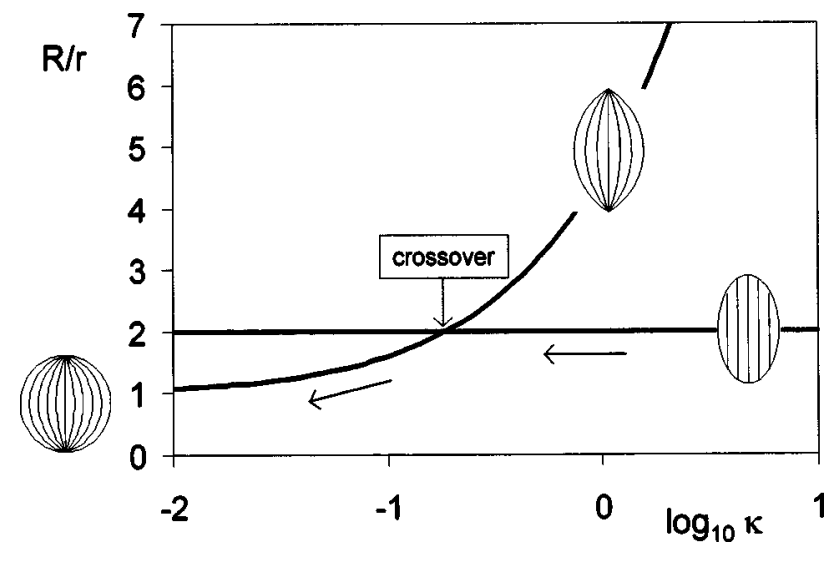

FIG. 7. Aspect ratio $R / r$ of a tactoid as a function of the dimensionless elastic stiffness $\kappa$. Horizontal curve-homogeneous director field with $\omega=1$, monotonically increasing curve-bipolar director field. Indicated is the transition between the bipolar director field, stable at small $\kappa$, to the homogeneous director field at large $\kappa$. The minuscule jump in the aspect ratio at the transition cannot be seen on the scale of the figure. 
strength of $\omega=1$. We have seen that provided $\kappa$ is sufficiently large, a homogeneous director field is always preferred, in which case the aspect ratio of the droplets does not depend on their size. For $\omega=1$, the theory predicts the most probable aspect ratio of $R / r=2$ to be a constant of $\kappa$ if $\kappa$ $>0.1843$. Consequently, the aspect ratio does not change with increasing drop size (decreasing $\kappa$ ), until the crossover to the bipolar structure is reached at $\kappa=0.1843$. There, the aspect ratio jumps from the value of 2 to a value of 1.993, in other words, for $\omega=1$ the droplet shape changes suddenly from an elongated spheroid to an elongated spindle of slightly smaller aspect ratio. Below $\kappa=0.1843$, the aspect ratio of the drops decreases with decreasing $\kappa$. It approaches the limiting value of unity for very large droplets, as $\kappa \rightarrow 0$.

The magnitude of the discontinuous change of the elongation of the droplets turns out to be a (nonmonotonic) function of $\omega$. However, as we already anticipated in Sec. III, the jump remains modest for all values of $\omega$ and is typically well below the maximum of about $12 \%$ that we find for large $\omega$ (results not shown).

Although our theory suggests the director-field and shape transformation to be discontinuous, this need not be the case in reality. In the following section, we speculate on a mechanism how this transformation could take place continuously, but not before first summarizing our findings.

\section{DISCUSSION AND CONCLUSIONS}

In conclusion, tactoids do not necessarily have a bipolar director field. If of sufficiently small size, their director field should be uniform. Presuming the surface tension is accurately represented by the form of Eq. (2), the precise shape of such small tactoids then solely depends on a dimensionless anchoring strength $\omega$. They are prolate spheroidal if $0<\omega$ $\leqslant 1$, and spindlelike with sharp ends if $\omega>1$. Large drops, on the other hand, prefer a bipolar director field, with a shape that is not rounded but spindlelike. The aspect ratio of bipolar tactoids decreases as a function of the volume. According to our calculations, bipolar drops can only become truly spherical in the limit of infinite volume.

If the anchoring strength is small, the transformation from a uniform to a bipolar director field occurs when the linear dimension $V^{1 / 3}$ of the droplet is of the order of the "extrapolation length" $l \equiv K / \tau \omega$ of the isotropic-nematic interface [36] and of the order of $l \omega^{1 / 6}$ if the anchoring strength is large. Our main conclusion, therefore, is that elongated tactoids postpone the crossover to a bipolar structure to a much higher droplet volume than spherical ones.

For values of $\omega$ not very much larger or smaller than unity, we estimate that for the usual thermotropic liquid crystals the director-field and shape transformation occurs when the droplets are of the order of one-tenth of a micron [29], which is why for this type of system the crossover is difficult to observe (except perhaps with $\mathrm{x}$-ray microdiffraction techniques [47]). This should not be so for lyotropic nematic tactoids on account of their much lower interfacial tension. For this type of system, the crossover size is likely to be in the micrometer range. Lacking any systematic experimental investigations of both the shape and the structure of the tac-

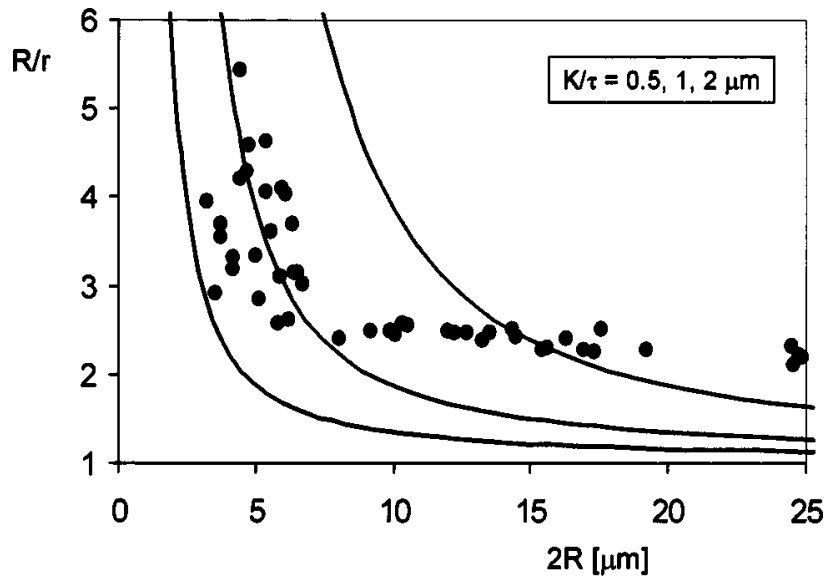

FIG. 8. Aspect ratio $R / r$ versus the length $2 R$ of nematic tactoids formed in aqueous dispersions of fd virus and the polymer dextran. The filled circles are the experimental data of Dogic [48], the drawn lines represent the theoretical results presuming a bipolar director field for three values of the length $K / \tau=0.5,1,2 \mu \mathrm{m}$ (from bottom to top).

toids, a comprehensive comparison of our theoretical predictions with experimental observations has to be left for future work.

As far as we are aware, the only experimental data that allow for a meaningful comparison with our theory are those of Dogic [48], who measured the dimensions of nematic tactoids that may (under the right conditions) be found in aqueous mixtures of the filamentous fd virus and the polysaccharide dextran. The presence of the polysaccharide induces an isotropic-smectic transition in the virus solution, the initial kinetics of which involve the formation of metastable nematic tactoids. Details of the experiments can be found in Ref. [4]. The results of the measurements are given in Fig. 8, together with theoretical predictions we obtained for bipolar tactoids. Theoretical curves are given for various values of the length $K / \tau$, which in the present description is the single adjustable parameter for the bipolar director-field configuration. The scatter in the data makes it difficult to fix this parameter accurately, but a value between 1 and $2 \mu \mathrm{m}$ seems to produce the best overall agreement. Clearly, theory and experiment agree only qualitatively, but this should not be surprising in view of the approximate nature of the theory and the scatter in the data.

A logical conclusion that one might draw from Fig. 8 is that the range of tactoid sizes sampled is not large enough to include the very smallest ones that have crossed over from a bipolar to a homogeneous director-field configuration. Indeed, there is no clear indication in the data of a levelling off of the aspect ratio of the drops at the lower end of the droplet sizes, as one would expect from the theory. Tentatively assuming the theory to be accurate all the way down to the smallest of droplets observed [49], we have to concede that the anchoring strength $\omega$ must be greater than about 6 for the theory to be consistent with the observations. This is obviously much larger than the theoretical values between 0.5 and 1.5 [9-12]. Note, however, that if the anchoring strength were as predicted to have a value around unity, tactoids with 


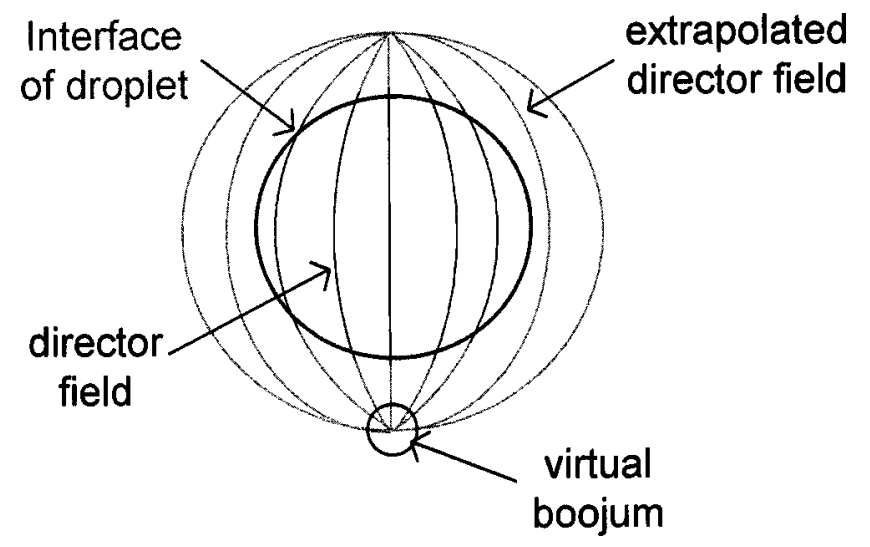

FIG. 9. Schematic representation of a nematic droplet with a quasibipolar director field that is intermediate between a true bipolar and a homogeneous director field. Indicated is one of the two virtual boojums, which are point defects in a director field extrapolated beyond the boundaries of the droplet. If these virtual boojums move in onto the actual interface of the droplets, they become true boojums. If the virtual boojums move out into infinity, the director field becomes homogeneous.

aspect ratios greater than about 2 would not exist. Highly elongated tactoids have been observed in a variety of systems [5], suggesting that the anchoring strength must indeed be much greater than found theoretically.

In practice, the crossover from a homogeneous to a bipolar director field may be subtle, i.e., it need not be quite as abrupt as we find in this work [29,30,50]. It may well be that the director field changes continuously with increasing droplet size in a process where virtual boojum defects gradually move in toward the surface. Virtual boojums are boojums in a director field extrapolated beyond the boundaries of the nematic droplet $[51,52]$. See Fig. 9. The larger the distance between the virtual boojums, the more homogeneous the actual director field. Polarization microscopic images of tactoids with real and quasibipolar director fields are probably quite similar and difficult to distinguish. Clearly, the issue calls for a more detailed study.

Although we have given arguments in favor of the equalconstant approximation, it does introduce an error that is $a$ priori difficult to estimate the impact of. A scaling analysis along the lines of that presented in Sec. III suggests that our conclusions are not radically altered if we allow the splay and bend elastic constants not to be equal. Indeed, if we set $\kappa_{S} \equiv K_{1} / \tau V^{1 / 3}$ and $\kappa_{B} \equiv K_{3} / \tau V^{1 / 3}$, we find that the crossover from the homogeneous to bipolar director field occurs either when $\omega \approx \kappa_{S}+\kappa_{B} \ll 1$ or when $\omega^{5 / 6} \approx \kappa_{S}+\kappa_{B} \omega^{-1} \gg 1$, depending on the anchoring strength. (Here, we have again dropped unknown constants of proportionality.) It follows that only in the regime where $\omega \gg 1$ and $1 \ll \kappa_{S} \ll \kappa_{B} \omega^{-1}$, we expect a change in scaling behavior of the aspect ratio of the drops because the contribution of the bend elasticity is in that case no longer negligible.

Another moot point is the stability of the twisted bipolar configuration, the possibility of which we have ignored completely. Such configurations have indeed been observed in thermotropic systems [13] and were investigated theoreti- cally by Williams [37]. According to Williams, tangentially anchored, bipolar nematic droplets of spherical shape crossover to a twisted configuration if $K_{1} \geqslant K_{2}+0.431 K_{3}$. In the twisted configuration, the splayed director field is partly replaced by a combined twisted and bent deformation. As a result, this configuration becomes energetically favorable if the splay elastic constant is sufficiently large in comparison with the other two (bulk) elastic constants. Because the splay elastic deformation is much smaller in elongated bipolar tactoids than in spherical ones, we should expect the twisted configuration to become less probable, the more elongated the tactoids are.

As a final remark, we note that the director-field and shape transformation of nematic droplets described in this paper should have a bearing on the rate of nucleation of the nematic phase in dispersions of elongated colloidal particles. According to classical nucleation theory, the free-energy barrier to nucleation is a function of the concentration of the nucleating nematic, the interfacial tension and the difference between the chemical potentials of the particles in the parent isotropic phase and in the nematic phase [53]. This should be more or less accurate if the quench is sufficiently deep for the critical nuclei to be so small that a homogeneous director field is preferred. In addition, the anchoring strength $\omega$ should not be too large for the critical nuclei to be more or less spherical. If the quench is shallow, however, or if the anchoring strength is large, the situation should be quite different and in fact much more complex. Depending on the quench depth, the nucleation barrier could in that case also depend on the anchoring strength and/or on the Frank elasticity of the critical nuclei. We intend to investigate the implications of these issues in the near future.

\section{ACKNOWLEDGMENTS}

We thank Jan Groenewold (TU Delft, The Netherlands) for valuable discussions and Thijs Michels (TU Eindhoven, The Netherlands) for a critical reading of the manuscript. We are especially indebted to Zvonimir Dogic (IFF Jülich, Germany) for discussions and for providing us with the (unpublished) experimental data.

\section{APPENDIX A: HOMOGENEOUS DROPS}

If the director field is homogeneous, the free energy of a droplet is given by Eq. (2). We seek to minimize this free energy with respect to the shape of the droplet at a fixed volume. In this appendix, we first consider two plausible families of droplet shape and minimize for these the free energy with respect to their aspect ratio. Next, we apply the Wulff construction [45] to Eq. (2) and obtain the shape known to represent that of the absolutely lowest free energy [7]. Virga recently discussed aspects of the results of the same calculation in Ref. [8] (and in work cited therein), but left out many of the details of the calculations. It seems justified, therefore, to present the full Wulff analysis in this appendix.

Let the director field of the droplet be along the $z$ axis. Assuming axial and inversion symmetry, the height of the 
droplet $0 \leqslant z(\rho) \leqslant R$ is a function only of the distance $\rho$ in the radial direction of a cylindrical coordinate system. The volume of the entire droplet is then given by $V=\int_{V} d^{3} \mathbf{r}$ $=4 \pi \int_{0}^{r} d \rho z(\rho) \rho$ and the surface area of the drop by $A$ $=\int_{A} d^{2} \mathbf{r}=4 \pi \int_{0}^{r} d \rho \rho \sqrt{1+\left[z^{\prime}(\rho)\right]^{2}}$, with $r$ the as yet unknown maximum radius of the droplet in the radial direction and $z^{\prime}=d z / d \rho$. The effective area involved in the nonplanar surface anchoring reads $A_{w} \equiv \int_{A} d^{2} \mathbf{r}(\mathbf{q} \cdot \mathbf{n})^{2}$ $=4 \pi \int_{0}^{r} d \rho \rho / \sqrt{1+\left[z^{\prime}(\rho)\right]^{2}}$.

The free energy of the drop is given by $F=F_{S}=\tau A$ $+\tau \omega A_{w}$. In order to render the free-energy invariant to the droplet volume we define $\widetilde{F} \equiv F / \tau V^{2 / 3}$, which is a function of the aspect ratio $R / r$ of the drops only. That this must be so is easily seen by making dimensionless the integration variable, $x \equiv \rho / r$, as well as the height function of the droplet, $\xi(x)$ $\equiv z(\rho) / R$. Inserting $\xi(x)=\sqrt{1-x^{2}}$, we get for the prolate ellipsoid with reciprocal aspect ratio $\varepsilon \equiv r / R<1$

$$
\widetilde{F}_{S}=\left(\frac{9 \pi \varepsilon^{2}}{2}\right)^{1 / 3}\left[\frac{\arccos \varepsilon}{\varepsilon \sqrt{1-\varepsilon^{2}}}+1+\omega \frac{\varepsilon^{2}}{1-\varepsilon^{2}}\left(\frac{\arccos \varepsilon}{\varepsilon \sqrt{1-\varepsilon^{2}}}-1\right)\right],
$$

while for the tactoid shape defined by the circle section contour $\xi(x)=\sqrt{1-\left(1-\varepsilon^{2}\right) x-\varepsilon^{2} x^{2}}$, we find

$$
\widetilde{F}_{S}=\left(\frac{4 \pi}{3}\right)^{1 / 3}\left[\frac{3\left(1+\varepsilon^{2}\right)^{2}(2+\omega)\left[\varepsilon-\left(1-\varepsilon^{2}\right) \arctan \varepsilon\right]-4 \omega \varepsilon^{3}}{\left(1+\varepsilon^{2}\right)\left(3\left(1+\varepsilon^{2}\right)^{2}\left[\varepsilon-\left(1-\varepsilon^{2}\right) \arctan \varepsilon\right]-4 \varepsilon^{3}\right)^{2 / 3}}\right],
$$

again for $\varepsilon<1$. To determine the optimal aspect ratio, the free energies Eqs. (A1) and (A2) have to be minimized with respect to $\varepsilon$. The resulting implicit equations for $\varepsilon$ are not reproduced here. We have not been able to find an analytical solution to these equations except in the limits $\varepsilon \rightarrow 1$ and $\varepsilon$ $\rightarrow 0$. The limiting solutions we obtained for $\varepsilon \rightarrow 1$ and $\varepsilon$ $\rightarrow 0$ confirm the scaling results of Sec. III.

An absolute minimum free-energy shape may be gotten by means of the Wulff construction [45]. In the Wulff construction, one draws a polar plot of the interfacial tension. Each point on this cylindrically symmetrical surface is connected to the center of the coordinate system by a radial vector. At the tip of each radial vector, a plane is defined perpendicular to it. The convex envelope of these planes gives the equilibrium droplet shape, i.e., points belong to the droplet if they can be reached from the center of the coordinate system without crossing any of the perpendicular planes. See, e.g., Refs. [7], [8] for a discussion of the Wulff construction in the context of liquid-crystal droplets.

Our problem is cylindrically symmetric and therefore effectively two dimensional. Focusing on the positive $z-\rho$ plane in the cylindrical coordinate system, the line perpendicular to the radial vector of an arbitrary point on the polar plot of the interfacial tension is described by

$$
z(\rho, \theta) \lambda^{-1}=-\rho \lambda^{-1} \tan \theta+\frac{1+\omega \cos ^{2} \theta}{\cos \theta},
$$

with $\theta$ the angle between the radial vector and the $z$ axis and $\lambda$ a length fixed by the volume of the droplet. (Note that we have absorbed the interfacial tension $\tau$ into $\lambda$.) Each point on the polar plot of the interfacial tension generates such a line. To determine the envelope of these lines, we minimize $z(\rho, \theta) \geqslant 0$ with respect to the angle $0 \leqslant \theta \leqslant \pi / 2$ for each $\rho$. Putting $x \equiv \sin \theta$, we thus have for the equilibrium drop profile

$$
z\left(\rho \lambda^{-1}\right) \lambda^{-1}=\frac{1+\omega\left(1-x^{2}\right)-x \rho \lambda^{-1}}{\sqrt{1-x^{2}}},
$$

with $x$ the solution of

$$
\omega x^{3}+(1-\omega) x-\rho \lambda^{-1}=0 .
$$

This cubic equation has at least one real solution for $0 \leqslant x$ $\leqslant 1$, depending on the dimensionless quantities $\omega$ and $\rho \lambda^{-1}$. We ascertain ourselves that the physically relevant solution chosen indeed represents the minimum $\rho$ for a given $z$.

By straightforward algebra, it follows that $z(0) \lambda^{-1}=1$ $+\omega$ if $0 \leqslant \omega \leqslant 1$ and $z(0) \lambda^{-1}=2 \sqrt{\omega}$ if $\omega \geqslant 1$, while $z(1) \lambda^{-1}=0$ for all $\omega$. This fixes the aspect ratio $R / r$ $=z(0) \lambda^{-1}$ of the drops because $\rho \lambda^{-1}=1$ for $x=1$. Furthermore, the tip angle as defined in the main text is given by the quantity $\arctan \left|z^{\prime}(0)^{-1}\right|$ with $z^{\prime}(0)=\lim _{\rho \rightarrow 0} d z / d \rho=0$ if 0 $\leqslant \omega \leqslant 1$ and $z^{\prime}(0)=-\sqrt{\omega-1}$ if $\omega>1$, where we note that $x$ is a function of $\rho$ through Eq. (A5).

Finally, after insertion of the droplet profile Eq. (A4) into the expressions for the volume $V$, and those for the areas $A$ and $A_{w}$, we find for the dimensionless free energy

$$
\widetilde{F}_{S}=3\left(\frac{4 \pi}{105}\left(35+35 \omega-7 \omega^{2}+\omega^{3}\right)\right)^{1 / 3},
$$

if $0 \leqslant \omega \leqslant 1$ and

$$
\widetilde{F}_{S}=3\left(\frac{32 \pi}{15} \omega^{1 / 2}\left(1+\frac{1}{7 \omega}\right)\right)^{1 / 3},
$$

if $\omega>1$, where we have eliminated the scale factor $\lambda$.

\section{APPENDIX B: BIPOLAR DROPS}

For droplets with a bipolar director field, the optimal shape cannot be straightforwardly calculated from a Wulff type of construction. (See, however, [52] for a generalized 
Wulff construction in the context of Langmuir layers.) For simplicity, we therefore prescribe plausible shapes and director-field configurations of the drops and optimize their aspect ratio. For the droplet shapes, we again assume the ellipsoid and spindle shapes introduced in the Appendix A. The surface free energies $\widetilde{F}_{S}$ for these shapes are given by Eqs. (A1) and (A2) in terms of the reciprocal aspect ratio $\varepsilon$ $=r / R$, with $\omega$ set equal to zero for we now impose a planar alignment of the director field to the droplet's interface. As before, $r$ denotes the half length of the minor axis and $R$ that of the major axis of the droplets. The total free energy $\widetilde{F}$ is now given by the sum of $\widetilde{F}_{S}$ and $\widetilde{F}_{E} \equiv \kappa F_{E} / K V^{1 / 3}$.

To calculate $\widetilde{F}_{E}$ of the prolate ellipsoid tactoids, we presume the director field to be accurately described by the normalized tangents to nested ellipsoids of revolution that cross nowhere but touch at the north and south poles along the main body axis (the $z$ axis). Ignoring possible twisted configurations, the director field $\mathbf{n}$ of a cylindrically symmetric bipolar director field may be written as $\mathbf{n}=\left[\mathbf{e}_{\rho}\right.$ $\left.+z^{\prime}(\rho) \mathbf{e}_{z}\right] / N$ with $N=\sqrt{1+z^{\prime}(\rho)^{2}}$ a normalization, $\mathbf{e}_{\rho}$ is the unit vector along the radial direction, and $\mathbf{e}_{z}$ that along the main symmetry axis of the droplet; $z(\rho)$ describes the shape of the nested ellipsoids with $\rho$ the radial coordinate. We focus on the positive half plane, $0 \leqslant z(\rho) \leqslant R$ and $0 \leqslant \rho \leqslant r$, and find for the director field $\mathbf{n}=\mathbf{n}(\rho, z)=\left[-\rho z R^{-2} \mathbf{e}_{\rho}+(1\right.$ $\left.\left.-z^{2} R^{-2}\right) \mathbf{e}_{z}\right] / N$ with $N=\sqrt{\rho^{2} z^{2} R^{-4}+\left(1-z^{2} R^{-2}\right)^{2}}$ again the normalization. The integrations of Eq. (3) and of the volume $V=4 \pi \int_{0}^{r} d \rho z(\rho) \rho$ are now straightforwardly performed, to give

$$
\widetilde{F}_{E}=\kappa\left(\frac{\pi}{6 \varepsilon}\right)^{2 / 3}\left(\varepsilon^{2}-2 \ln \frac{1}{2} \varepsilon+\frac{\operatorname{arcsech} \varepsilon}{\sqrt{1-\varepsilon^{2}}}\left(\varepsilon^{4}+13 \varepsilon^{2}-2\right)\right),
$$

provided $\varepsilon \leqslant 1$. The equilibrium shape can be found by minimizing $\widetilde{F}_{E}+\widetilde{F}_{S}$ with respect to $\varepsilon$ and yields an implicit expression for $\varepsilon(\kappa)$ not reproduced here.

For the spindle-shaped droplets, we choose a director field that is given by the (normalized) tangents to families of circle sections of revolution that cross at the poles $z= \pm R$. For this director field, the various gradients and integrals are the easiest to be performed in a bispherical coordinate system [37]. If we place the center of the bispherical coordinate system at $z=0$, the transformation from the cylindrical coordinates $(\rho, \phi, z)$ to the bipolar coordinates $(\xi, \eta, \phi)$ is given by $z / R=Z^{-1} \cos \xi$ and $\rho / R=Z^{-1} \sin \xi \sin \eta$, where $Z=1$ $+\sin \xi \cos \eta$.

At fixed $\phi$, the lines of constant $\eta$ are circles crossing the poles at $z= \pm R$, while the lines of constant $\xi$ orthogonal to those are circles too. The director field is in this representation given by $\mathbf{n}=\mathbf{e}_{\xi}$, with $\mathbf{e}_{\xi}$ the unit vector along the lines of constant $\eta$ and $\phi$. The ranges of the bispherical coordinates describing the droplet are given by $0 \leqslant \phi \leqslant 2 \pi, 0 \leqslant \xi \leqslant \pi$, and $0 \leqslant \eta \leqslant \eta_{0}$ with the bound $\eta_{0}=2 \arctan \varepsilon$ fixing its reciprocal aspect ratio $\varepsilon=r / R$.

To transform the various surface and volume integrals, we need the metric elements $h_{\xi}=R Z^{-1}, h_{\eta}=R Z^{-1} \sin \xi$, and $h_{\phi}=R Z^{-1} \sin \xi \sin \eta$. The volume of the droplet is given by $V=\int_{0}^{2 \pi} d \phi \int_{0}^{\pi} d \xi \int_{0}^{\eta_{0}} d \eta h_{\phi} h_{\xi} h_{\eta}$ and its surface area by $A$ $=\int_{0}^{2 \pi} d \phi \int_{0}^{\pi} d \xi h_{\phi} h_{\xi}$, where for the latter, the metric elements $h_{\phi}$ and $h_{\xi}$ are evaluated at $\eta=\eta_{0}$. These integrals are readily performed.

To calculate the Frank elastic energy Eq. (2), various gradients of the director field have to be evaluated. For the bispherical director field, they reduce to $(\boldsymbol{\nabla} \cdot \mathbf{n})^{2}$ $=4 R^{-2} \cot ^{2} \xi, \quad[\mathbf{n} \cdot(\boldsymbol{\nabla} \times \mathbf{n})]^{2}=0, \quad[\mathbf{n} \times(\boldsymbol{\nabla} \times \mathbf{n})]^{2}$ $=R^{-2} \sin ^{2} \eta$, and $\boldsymbol{\nabla} \cdot(\mathbf{n} \boldsymbol{\nabla} \cdot \mathbf{n}+\mathbf{n} \times(\boldsymbol{\nabla} \times \mathbf{n})]=2 R^{-2} \cot ^{2} \xi$. Note that the first (splay) and last (saddle-splay) terms are identical up to a factor of 2, which is the reason why in Sec. II, we absorbed $K_{24}$ into $K_{1}$, i.e., put $K_{1} \equiv K_{1}-K_{24}$. Apparently, the saddle-splay term stabilizes the bipolar director field by reducing the free-energy cost of the splay deformation. (A similar effect is seen in cylindrical geometries [54].) The remaining terms give for the dimensionless free energy in the equal-constant approximation

$$
\widetilde{F}_{E}=6 \kappa\left(\frac{4 \pi}{3}\right)^{2 / 3} \frac{\frac{7}{4}\left[\varepsilon-\left(1-\varepsilon^{2}\right) \arctan \varepsilon\right]-\varepsilon \arctan ^{2} \varepsilon}{\left(3(1+\varepsilon)^{2}\left[\varepsilon-\left(1-\varepsilon^{2}\right) \arctan \varepsilon\right]-4 \varepsilon^{3}\right)^{1 / 3}},
$$

which, after adding the surface free energy $\widetilde{F}_{S}$, we minimize to obtain the optimal $\varepsilon$. Again, this does not produce an explicit expression giving $\varepsilon$ as a function of $\kappa$, only an implicit one that we again do not reproduce here. This equation we solved analytically in the limits $\varepsilon \rightarrow 1$ and $\varepsilon \rightarrow 0$, and by standard numerical methods to get the full $\kappa$ dependence of $\varepsilon$.
[1] J. D. Bernal and I. Fankuchen, J. Gen. Physiol. 25, 111 (1941).

[2] H. Zocher and C. Török, Kolloid-Z. 170, 140 (1960).

[3] J. D. Bernal and I. Fankuchen, Nature (London) 139, 923 (1937).

[4] Z. Dogic and S. Fraden, Philos. Trans. R. Soc. London, Ser. A 359, 997 (2001).

[5] A. S. Sonin, Colloid J. USSR 60, 129 (1998).

[6] C. Herring, Phys. Rev. 82, 87 (1951).

[7] S. Chandrasekhar, Mol. Cryst. 2, 71 (1966).

[8] E. G. Virga, Variational Theories for Liquid Crystals (Chapman and Hall, London, 1994).
[9] Z. Y. Chen and J. Noolandi, Phys. Rev. A 45, 2389 (1992).

[10] S.-M. Cui, O. Akcakir, and Z. Y. Chen, Phys. Rev. E 51, 4548 (1995).

[11] D. L. Koch and O. G. Harlen, Macromolecules 32, 219 (1999).

[12] P. van der Schoot, J. Phys. Chem. B 103, 8804 (1999).

[13] P. S. Drzaic, Liquid Crystal Dispersions (World Scientific, Singapore, 1995).

[14] H. Zocher and K. Jacobson, Koll. Beih. 28, 167 (1929).

[15] R. D. Williams, Rutherford Appleton Laboratory Report No. RAL-85-028, 1985 (unpublished).

[16] S.-D. Lee and R. B. Meyer, J. Chem. Phys. 84, 3443 (1986). 
[17] S. Itou, K. Tozaki, and N. Komatsu, Jpn. J. Appl. Phys., Part 1 30, 1230 (1991).

[18] V. G. Taratuta, A. J. Hurd, and R. B. Meyer, Phys. Rev. Lett. 55, 246 (1985).

[19] V. G. Taratuta, F. Lonberg, and R. B. Meyer, Phys. Rev. A 37, 1831 (1988).

[20] D. B. DuPré and R. W. Duke, J. Chem. Phys. 63, 143 (1975).

[21] A. J. Hurd, S. Fraden, F. Lonberg, and R. B. Meyer, J. Phys. (Paris) 46, 905 (1985).

[22] S. Fraden, A. J. Hurd, R. B. Meyer, M. Cahoon, and D. L. D. Casper, J. Phys. (Paris), Colloq. 46, C3-85 (1985).

[23] S.-D. Lee and R. B. Meyer, Phys. Rev. Lett. 61, 2217 (1988).

[24] W. Chen, T. Sato, and A. Teramoto, Macromolecules 29, 4283 (1996).

[25] W. Chen, T. Sato, and A. Teramoto, Macromolecules 31, 6506 (1998)

[26] W. Chen, T. Sato, and A. Teramoto, Macromolecules 32, 1549 (1999).

[27] W. Chen and D. G. Gray, Langmuir 18, 633 (2002).

[28] One simply balances an elastic distortion energy against a surface anchoring energy [13]. See also Sec. III. By a similar argument one finds that for droplets with a preference for homeotropic anchoring attain a homogeneous director field if sufficiently small. See, e.g., J. H. Erdmann, S. Zumer, and J. W. Doane, Phys. Rev. Lett. 64, 1907 (1990).

[29] W. Huang and G. F. Tuthill, Phys. Rev. E 49, 570 (1994).

[30] A. G. Kalugin and A. N. Golubyatnikov, Proc. Steklov Inst. Math. 223, 168 (1998).

[31] I. Vilfan, M. Vilfan, and S. Zumer, Phys. Rev. A 40, 4724 (1989).

[32] A. Rapini and M. J. Papoular, J. Phys. (Paris), Colloq. 30, C4-54 (1969)

[33] F. C. Frank, Discuss. Faraday Soc. 25, 19 (1958).

[34] C. Oseen, Trans. Faraday Soc. 29, 883 (1933).

[35] J. Nehring and A. Saupe, J. Chem. Phys. 54, 337 (1971).

[36] P. G. de Gennes and J. Prost, The Physics of Liquid Crystals (Clarendon, Oxford, 1993).
[37] R. D. Williams, J. Phys. A: Math. Gen. 19, 3211 (1986).

[38] We also investigated bipolar parabolas of revolution and found the free-energy difference between these and circle sections of revolution to be under $1 \%$ for all droplet volumes. For this reason, we do not discuss parabolas of revolution and take the circle section of revolution as representative for the class of elongated, pointed droplets.

[39] After submission of the manuscript our attention was drawn on a paper by A. V. Kaznacheev, M. M. Bogdanov, and S. A. Taraskin, JETP 95, 57 (2002). Their study focuses on the shape of bipolar nematic droplets larger than $\approx 20 \mu \mathrm{m}$, away from the crossover region.

[40] T. Odijk, Liq. Cryst. 1, 553 (1986).

[41] T. Sato and A. Teramoto, Macromolecules 29, 4107 (1996).

[42] G. J. Vroege and T. Odijk, J. Chem. Phys. 87, 4223 (1987).

[43] D. Adrienko and M. P. Allen, Phys. Rev. E 65, 021704 (2002).

[44] We only explicitly consider spherical and elongated shapes, not toroidal or flat ones as these are (within our assumptions) structures of higher energy, where we note that oblate spheroid and lens-shaped droplets can become stable if $-1<\omega<0$ $[8,15,30,37]$.

[45] G. Wulff, Z. Kristallogr. 34, 449 (1901).

[46] E. Dubois-Violette and O. Parodi, J. Phys. (Paris), Colloq. 30, C4-57 (1969).

[47] O. Francescangeli, C. Ferrero, L. Lucchetti, F. Simoni, and M. Burghammer, Europhys. Lett. 59, 218 (2002).

[48] Z. Dogic (unpublished).

[49] The accuracy of our theory should deteriorate when the droplets become of the order of the size of the nematogens, which is about $1 \mu \mathrm{m}$ for fd virus. A continuum description then loses its validity.

[50] P. K. Chan and A. D. Rey, Liq. Cryst. 23, 677 (1997).

[51] S. Rivière and J. Meunier, Phys. Rev. Lett. 74, 2495 (1995).

[52] J. Rudnick and R. Bruinsma, Phys. Rev. Lett. 74, 2491 (1995).

[53] R. P. Sear (unpublished).

[54] R. J. Ondris-Crawford, G. P. Crawford, S. Zumer, and J. W. Doane, Phys. Rev. Lett. 70, 194 (1993). 